\title{
Metagenomic Next-generation Sequencing of Cerebrospinal Fluid for the Diagnosis of Central Nervous System Infections: A Multicentre Cohort Study
}

\section{Siyuan Fan}

Peking Union Medical College Hospital

\section{Xiaojuan Wang}

henan Provincial People's Hospital

Yafang Hu

Southern Medical University Nanfang Hospital

Jingping Shi

Nanjing Brain Hospital

\section{Yueli Zou}

Second Hospital of Hebei Medical University

\section{Weili Zhao}

Affiliated Hospital of Chifeng University

\section{Xiaodong Qiao}

Affiliated Hospital of Chifeng University

\section{Chunjuan Wang}

Shandong Provincial Hospital

Jerome H. Chin

NYU Langone Health

Lei Liu

Beijing Tongren Hospital

\section{Lingzhi Qin}

Henan Pronvicial People's Hospital

\section{Shengnan Wang}

Southern Medical University Nanfang Hospital Hongfang Li

Affiliated Hospital of Jining Medical University

\section{Wei Yue}

Tianjin Huanhu Hospital

\section{Weihe Zhang}

China-Japan Friendship Hospital 


\section{Xiaohua Li}

Affiliated Hospital of Inner Mongolia Medical College

\section{Ying Ge}

Peking Union Medical College Hospital

\section{Honglong Wu}

BGI

\section{Weijun Chen}

University of the Chinese Academy of Sciences

\section{Yongjun Li}

Vision Medicals Co., Ltd

\section{Shiying Li}

North China University of Science and Technology

\section{Yihan Wu}

Inner Mongolia People's Hospital

\section{Gaoya Zhou}

Brain Hospital of Hunan Province

\section{Zheng Liu}

\section{Xuanwu Hospital}

\section{Yushun Piao}

Shenzhen Baoan Shiyan People's Hospital

\section{Jianzhao Zhang}

Capital Institute of Pediatrics

\section{Changhong Ren}

Beijing Children's Hospital

\section{Li Cui}

Jinin University First Hospital

Caiyun Liu

Jinin University First Hospital

\section{Haitao Ren}

Peking Union Medical College Hospital

\section{Yanhuan Zhao}

Peking Union Medical College Hospital

\section{Shuo Feng}

Capital Institute of Pediatrics

\section{Haishan Jiang}

Nanfang Hospital

\section{Jiawei Wang}

Beijing Tongren Hospital

\section{Hui Bu}


Second Hospital of Hebei University

\section{Shougang Guo}

Shandong Provincial Hospital

\section{Bin Peng}

Peking Union Medical College Hospital

\section{Liying Cui}

Peking Union Medical College Hospital

\section{Wei Li}

Henan Provincial People's Hospital

Hongzhi Guan ( $\sim$ guanhz@263.net )

\section{Research article}

Keywords: encephalitis, meningitis, metagenomic, next-generation sequencing, pathogen

Posted Date: November 15th, 2019

DOl: https://doi.org/10.21203/rs.2.17334/v1

License: (c) (i) This work is licensed under a Creative Commons Attribution 4.0 International License. Read Full License 


\section{Abstract}

Background: Infectious encephalitis and meningitis are often treated empirically without identification of the causative pathogen, even if comprehensive conventional diagnostic technologies are applied. Metagenomic next-generation sequencing (mNGS) is a high throughput technology that enables the detection of pathogens independent of prior clinical or laboratory information.

Methods: The present study was a multicentre prospective evaluation of mNGS of cerebrospinal fluid (CSF) for the diagnosis of suspected central nervous system infections in China, where routine PCR was not widely used.

Results: A total of 276 patients were enrolled in this study between Jan 1, 2017 and Jan 1, 2018. Identification of an aetiologic pathogen in CSF by mNGS was achieved in 101 patients (36.6\%). mNGS detected 11 bacterial species, 7 viral species, 2 fungal species, and 2 parasitic species. The leading positive detections were Mycobacterium tuberculosis (14), Listeria monocytogenes (8), Brucella (7), varicella-zoster virus (17), herpes simplex virus 1 (12), Epstein-Barr virus (12), and Cryptococcus neoformans (7). False positives occurred in $12(4.3 \%)$ patients with bacterial infections known to be widespread in hospital environments. False negatives occurred in $16(5.8 \%)$ patients and included bacterial, viral and fungal aetiologies.

Conclusions: This study shows that mNGS of CSF is a powerful diagnostic method to identify the pathogen for many central nervous system infections. The result of mNGS should be interpreted with clinical information sometimes.

\section{Abbreviations}

mNGS: Metagenomic next-generation sequencing; CSF: cerebrospinal fluid; CNS: central nervous system; HSV1: herpes simplex virus 1; VZV: varicella zoster virus; NTC: 'no-template' control; RPM: reads per million; SSRN: species-specific read number; MTC: Mycobacterium tuberculosis complex; IQR: interquartile range; EBV: Epstein-Barr virus; CMV: cytomegalovirus.

\section{Introduction}

Infectious encephalitis and meningitis are major contributors to the neurological global burden of disease [1-4]. Numerous microorganisms, including bacteria, viruses, fungi, and parasites, can cause encephalitis and meningitis in immunocompetent or immunocompromised hosts; but the clinical manifestations of many infections are non-specific. Using comprehensive conventional diagnostic technologies, microbiological detection of the pathogen is achieved in only $50-80 \%$ of cases [5-8]. The inability to identify the infectious aetiology of encephalitis and meningitis often results in delayed, inadequate, and/or inappropriate treatment. 
Metagenomic next-generation sequencing (mNGS) is a novel tool that allows for the simultaneous and independent sequencing of thousands to billions of DNA fragments [9]. Cerebrospinal fluid (CSF) is particularly suitable for NGS due to its sterility in healthy individuals. Compared with traditional individual target-specific tests, mNGS can identify pathogens without the input of clinical predictors or prior laboratory results. Several recent studies have demonstrated the capability of mNGS of CSF to identify known and unsuspected pathogens and to discover new microorganisms [10-18]. mNGS of CSF is being increasingly utilized in routine clinical settings for the rapid diagnosis of central nervous system infections. However, most published studies are retrospective case reports or case series [11-17,19-24], and thus, large prospective studies are needed to demonstrate the clinical impact and cost-effectiveness of $\mathrm{mNGS}$ for the diagnosis of meningitis and encephalitis. We undertook a multicentre prospective study to comprehensively evaluate the performance of mNGS of CSF for the diagnosis of central nervous system (CNS) infections compared to conventional microbiological methodologies.

\section{Materials And Methods}

\subsection{Participants and study design}

This study was a multicentre prospective cohort assessment of the mNGS of CSF for the diagnosis of suspected infectious encephalitis or meningitis. The participating sites were 20 hospitals located in 10 provinces/municipalities in China. Each hospital is a member of the Beijing Encephalitis Group. Adult patients were eligible for inclusion in the study if they presented with clinical manifestations consistent with either encephalitis or meningitis (Table 1) and if standard diagnostic examinations (Supplementary Table 1) failed to identify an etiological cause within 3 days (PCR tests for viruses detection were not obligatory prior to enrolment). Exclusion criteria are shown in Table 1.

mNGS were conducted on all CSF specimens. Relevant conventional microbiological studies (e.g. staining, culture, polymerase chain reaction [PCR], serology) were arranged respectively according to the clinical manifestations and/or the results of mNGS. Conventional microbiological studies were considered the gold standard according to relevant guidelines and/or consensus [2,25-27] to classify the results of mNGS as true-positive, false-positive, and false-negative. Detected pathogens were classified as etiologic pathogens if the major clinical manifestations of the patient were consistent with that pathogen. All patients were treated based on the results of conventional microbiological testing (or empirically if results were negative) according to the latest clinical guidelines and/or consensus. Patients were followed for at least 30 days to determine the final diagnosis. Demographic data, medical history, laboratory test results (including all conventional microbiological tests), neuroimaging findings, medical therapy, and response to treatment were collected prospectively. Patients enrolled from Jan 1, 2017 to Jan 1, 2018 were included in the final analyses.

This study was approved by the institutional review board of Peking Union Medical College Hospital (no. JS-890). Written informed consent was obtained from each patient or their legal surrogate prior to 
enrolment. Written informed consent for the use of individual's data was also obtained. The study was performed in accordance with the Declaration of Helsinki.

\section{- mNGS of CSF}

CSF samples were collected according to standard sterile procedures, snap-frozen, and stored at $-20^{\circ} \mathrm{C}$ until they were delivered to the sequencing centre. Because reverse transcription was not performed to prepare DNA libraries, RNA viruses were not investigated in this study. mNGS of the CSF samples was performed using a standard flow that has been successfully used to detect herpes simplex virus 1 (HSV1), HSV2, varicella zoster virus (VZV), Listeria monocytogenes, Brucella, and Taenia solium [12-15].

DNA was extracted from $300 \mu \mathrm{L}$ of CSF and negative 'no-template' controls (NTCs). Sequencing was performed on the BGISEQ-100 platform with an average of 20 million total reads obtained for each sample. The qualified reads were mapped to the human reference genome using the Burrows-Wheeler Aligner to remove human sequences. The remaining reads were aligned to the database of annotation, which includes the NCBI microbial genome database (ftp://ftp.ncbi.nlm.nih.gov/genomes/) to detect pathogens. The sequencing data was analysed in terms of the numbers of raw reads, non-human reads, and reads aligned to the microbial genome database as well as species-specific reads (genus-specific reads for Mycobacterium tuberculosis and Brucella), reads per million (RPM), and genome coverage (\%). The results of mNGS were available in no more than $48 \mathrm{hrs}$.

\subsection{Criteria for positive results of mNGS of CSF samples}

To reduce the influence of potential contamination, we used the following criteria for positive results of CSF mNGS:

1) For extracellular bacteria, fungi (excluding Cryptococcus), and parasites, the result was considered positive if a species detected by mNGS had a species-specific read number (SSRN) $\geq 30$ (RPM $\geq 1.50$ ) that ranked among the top 10 for bacteria, fungi, or parasites. Organisms detected in the NTC or that were present in $\geq 25 \%$ of samples from the previous 30 days were excluded but only if the detected SSRN was $\geq 10$-fold than that in the NTC [28] or other organisms. Additionally, organisms present in $\geq 75 \%$ of samples from the previous 30 days were excluded.

2) For intracellular bacteria (excluding Mycobacterium tuberculosis and Brucella) and Cryptococcus, the result was considered positive if a species detected by NGS had a SSRN $\geq 10$ (RPM $\geq 0.50$ ) [13] that ranked among the top 10 for bacteria or fungi. Pathogens detected in the NTC or that were present in $\geq$ $25 \%$ of samples from the previous 30 days were excluded but only if the detected SSRN was $\geq 10$-fold than that in the NTC or other organisms. 
3) For viruses, Brucella, and Mycobacterium tuberculosis, the result was considered positive if a species (or genus for Mycobacterium tuberculosis [Mycobacterium tuberculosis complex, MTC] and Brucella) detected by NGS had a SSRN $\geq 3$ (RPM $\geq 0.15)[12,28]$. Pathogens detected in the NTC were excluded but only if the detected SSRN was $\geq 10$-fold than that in the NTC. In our previous clinical observations, there were a few cases without Mycobacterium tuberculosis infection which contained MTC-specific reads number of 1 in the mNGS results. To mitigate the possibility of false positives, we adopted the criteria of SSRN $\geq 3$ rather than SSRN $\geq 1[24]$ in this study.

The performance of the criteria were evaluated at the finally stage of the study, the original results of $\mathrm{mNGS}$ and/or clinical manifestations were used to guide the further testing of conventional microbiological studies.

\subsection{Statistical analysis}

All statistical analyses were conducted using Statistical Package for the Social Sciences (SPSS) version 17.0 and EXCEL 1810. Depending on their distribution, all data are expressed as medians with interquartile ranges (IQRs) or as means \pm standard deviation.

\section{Results}

\subsection{Characteristics of the study participants}

287 patients were screened for inclusion in this study (Fig. 1). 11 patients were initially thought to have CNS infections and mNGS was performed. However, these cases were ultimately excluded following the final diagnosis of a non-infectious disease. Of these 11 excluded patients, 10 had negative mNGS results, and 1 patient receiving immunosuppressive therapy was positive for BK polyomavirus. The final cohort included 276 patients in the study. 176 (63.8\%) were male and the median age was 42 years (IQR: 26-54 years). The median time from disease-onset to CSF sampling was 10 days (IQR: 5-25 days). The median white blood cell count in CSF was $80 / \mathrm{mm}^{3}$ (IQR: $19-220 / \mathrm{mm}^{3}$ ). The median CSF mononuclear cell count was $36 / \mathrm{mm}^{3}$ (IQR: $10-127 / \mathrm{mm}^{3}$ ). During a follow-up period of 30 days, nine patients died.

\subsection{Performance of mNGS for diagnosing CNS infections}

276 CSF samples were tested by mNGS and conventional microbiological studies. 122 samples were positive by mNGS (110 true positive, 12 false positive), 126 were positive by conventional microbiological tests, and 114 total positive results were considered 'aetiologic pathogens' (Table 2). All mNGS results were obtained in less than 48 hours and 101 CSF samples considered 'aetiologic pathogens' were positive by mNGS. 
Of the patients diagnosed by mNGS, $16.3 \%$ of infections were caused by bacterial, $15.2 \%$ by viruses, $2.9 \%$ by fungi, and $2.2 \%$ by parasites (Fig. 2 A).

In total, mNGS detected 11 bacterial species, of which $M$. tuberculosis (14 cases, $13.9 \%$ ) and $L$. monocytogenes (7.9\%) were the most common (Fig. 2B), 7 viral species (BK polyomavirus was not the etiologic pathogen), of which VZV (16.8\%) and HSV1 (11.9\%) were the most common, 2 fungal species, both of which were Cryptococcus (7.9\%), and 2 parasitic species, of which $T$. solium (5.0\%) was the most common. Nine co-infections with Epstein-Barr virus (EBV) (three with HSV1, two with Brucella, one with Cryptococcus, one with S. haemolyticus, one with P. aeruginosa, and one with M. tuberculosis), two coinfections with cytomegalovirus (CMV) (one with M. tuberculosis, and one with Cryptococcus), and one co-infection with BK polyomavirus (with HSV1) were detected. The EBV and BK polyomavirus did not appear to be consistent with the clinical manifestations in these two instances of co-infections.

\subsection{False positive results of CSF $\mathrm{mNGS}$}

In the present study, false positives occurred in $12(4.3 \%)$ patients and were primarily associated with bacterial infections ( $\mathrm{n}=12$; Table 2), including E. coli, E. faecium, A. baumannii, S. maltophilia, and $P$. aeruginosa, and a false positive for Brucella was also seen. Of note, the false-positive samples contained numerous other bacteria, that could be detected simultaneously by NGS. Using our proposed criteria, there were no false positives for viruses, fungi, or parasites. Although EBV was not the etiologic pathogen in most cases, it was present in the CSF of some patients. Additionally, there was some background contamination in most CSF samples (Supplementary Table 2) but these organisms did not meet the criteria for a positive result.

\subsection{False negative results of CSF mNGS}

In the present study, false negatives occurred in $16(5.8 \%)$ patients (Table 2 ) and were associated with bacterial, viral and fungal infections. The false negative cases of bacterial infection were all treated with antibiotics prior to sequencing. In the false negative cases of viral infection, 1 or 2 SSRNs were detected in the samples but did not satisfy the proposed criteria for a positive mNGS result. If the criteria for a positive result was relaxed to a SSRN $\geq 1$ (RPM $\geq 0.05$ ), there were no false negative cases of HSV1 or VZV or false positive cases of HSV1, HSV2 or VZV. In this study, if we adopted the alternate criteria SSRN $\geq 1$ (RPM $\geq 0.05$ ) for viruses and Mycobacterium tuberculosis, there would be additional potential false positives, including $30 \mathrm{EBV}, 7 \mathrm{CMV}$ and 5 Mycobacterium tuberculosis infections. It should be pointed out that the possibility of Mycobacterium tuberculosis infection in the 5 cases cannot be ruled out based on the clinical and paraclinical manifestations, because the conventional microbiological methods might fail to detect the Mycobacterium tuberculosis. Of note, there were three false negative cases of Cryptococcus infection. 


\subsection{Illustrating cases of the study}

Case 1: An old male with a medical history of gastric cancer presented with cognitive decline, psychiatric symptoms and seizures for 1 month. Brain MRI revealed a lesion in the left frontal lobe, and the first lumber puncture revealed no pleocytosis. Therefore, 'autoantibody-negative autoimmune encephalitis' was suspected with the negative results of the antibodies panel. After the treatment with steroids and IVIg, the symptoms aggravated, and the repeated brain MRI showed diffuse cortical lesions in the left hemisphere. After referred to our centre, NGS of CSF was performed and detected abundant HSV 1, which was confirmed with PCR. He was diagnosed with herpes simplex encephalitis and responded well to acyclovir.

Case 2: An old male presented with right eye pain, memory deficits and psychiatric symptoms. Brain MRI showed a lesion in the right mesial temporal lobe. TORCH panel of CSF was positive for CMV IgM. Encephalitis caused by CMV was suspected. However, VZV was detected with NGS of CSF and confirmed with PCR. He was diagnosed with VZV encephalitis.

Case 3: A young male experienced hyperpyrexia and headache for 3 times in the past 10 years. He recovered quickly after the treatment with antibiotics every time without identifying the exact pathogen. Mollaret meningitis was suspected by the local hospital. When he presented with hyperpyrexia, headache and drowsiness 1 months ago, NGS of CSF was performed and detected Streptococcus pneumoniae. Further examinations were performed for the cause of recurrent purulent meningitis. At last, cerebrospinal fluid rhinorrhoea was found out and fixed.

\section{Discussion}

The present study is aimed to assess the performance of mNGS for pathogen identification in a prospective cohort of patients with suspected CNS infections. This study represents the application of mNGS in the settings in which routine PCR was not widely used. Specifically, our study compared results of mNGS of CSF to conventional microbiological studies and proposed new criteria for validating a mNGS result as positive for therapeutic decision-making. Besides, this study provided a pathogen spectrum of infectious encephalitis or meningitis in China. Our results suggest that NGS can provide a quicker and more accurate aetiologic pathogen identification than conventional microbiological methods. However, patients in the present study were only eligible to be assessed by mNGS if conventional microbiological studies, e.g. routine bacterial stains and cultures, India ink preparation, serological tests, failed to identify an etiologic cause within 3 days. Thus, the application of CSF mNGS in the clinical setting of this study could be regarded as a quasi-first line method for diagnosing CNS infectious diseases. 
mNGS is a high-throughput sequencing technique without the requirement of prior information, allowing detection of unsuspected or novel organisms. Importantly, mNGS can detect unsuspected pathogens that clinicians may fail to consider because of atypical clinical manifestations. Many cases of neurological infections have been unexpectedly diagnosed by $\operatorname{mNGS}$ of CSF $[11,22,29,30]$ similar to the present study for the cases of L. monocytogenes, Brucella and T. solium [12-14]. In addition, as demonstrated in previous studies $[10,20,21]$ and in the present study for the case of encephalitis caused by Suid herpesvirus 1 [31], mNGS of CSF has the ability to identify novel aetiologies of CNS infections. Furthermore, NGS can detect unexpected co-infections that may guide appropriate targeted treatment. For example, we detected co-infections of CMV and Cryptococcus. In routine clinical practice, if conventional microbiological methods detect Cryptococcus, then no further tests for other microorganisms other than HIV are usually performed. Finally, mNGS of CSF may be an appropriate tool for ruling out a broad spectrum of potential CNS infectious diseases prior to concluding a final diagnosis of autoimmune diseases, such as autoantibody-negative autoimmune encephalitis.

Contamination of samples during specimen collection and/or processing is a major challenge when interpreting mNGS results. To reduce the potential influence of contamination, we defined strict criteria for positive mNGS results. The various types of contamination observed in the present study could be divided into two groups: (1) microorganisms commonly associated with background contamination that did not meet the criteria for a positive result (Supplementary Table 2) and (2) false positive detections that fulfilled our criteria for a positive mNGS result but were not consistent with the patients' clinical presentation and features. The contaminations derived primarily from the following sources: (1) laboratory practices (Parvovirus NIH CQV is a contaminant from silica column-based nucleic acid extraction kits) [32]; (2) reagents (Bradyrhizobium, Burkholderia and Ralstonia are common contaminants used in industrial ultrapure water systems) [33,34]; (3) environment (E. coli, P. aeruginosa, E. faecium and Torque teno virus are widespread pathogens in hospital environment) [35,36]; (4) skin or other body flora (P. acnes, M. globose, E. coli and S. epidermidis are widely associated with the human skin flora) $[37,38]$. False positive results are very likely to misguide treatment, and therefore, clinicians should be cautious when interpreting positive mNGS detection of extracellular bacteria or fungi that are widespread in hospital environments, especially when many species of bacteria are detected in a single NGS test. On the other hand, positive mNGS detection of viruses and parasites are not likely to be false positives.

Negative mNGS results do not necessarily exclude an infectious cause for the patient's illness. In the present study, false negative mNGS results occurred in $5.8 \%$ of cases and included bacterial, viral, and fungal infections. The prior use of antibiotics can affect the detection rate of bacteria. Low SSRN values ( 1 or 2 reads) can be seen in false negative results for virus detection indicating that other microbiological tests should be conducted to confirm a diagnosis when the SSRN is low for viruses. False negative cases have been reported for Cryptococcus due to fungal counts below the lower limit of detection for nucleic acid amplification tests $[39,40]$. The criteria of SSRN $\geq 3$ rather than SSRN $\geq 1$ might introduce false-negative cases for Mycobacterium tuberculosis infections. As a screening method, the pathogens detected by mNGS might provide clinical clues for further investigation even if the results do not fulfil the specified criteria for a positive result. 
Our results indicate that mNGS of CSF is a very useful test for the diagnostic evaluation of patients with suspected CNS infections. mNGS has already become a first-line laboratory method in the response to emerging infectious diseases and outbreaks of infectious diseases [41,42]. Moreover, increasing evidence provides a rationale for using mNGS as a first-line diagnostic test for chronic and recurring encephalitis and as a second-line test for acute encephalitis [23]. In the present study, more than one-third of patients were diagnosed by mNGS of CSF within $48 \mathrm{hrs}$, indicating that this technique can be extremely useful for rapid clinical decision-making. mNGS of CSF should be considered as a first-line test for acute CNS infections when (1) a patient is critically ill and requires prompt and precise therapy, (2) the clinical manifestations are non-specific and numerous target-specific tests are simultaneously applied to identify an infectious cause, 3 ) a broad spectrum of potential pathogens needs to be ruled out to diagnose a suspected autoimmune encephalitis, and (4) rare or novel pathogens are suspected for which standard target-specific tests are not available.

There are several limitations regarding the current use of mNGS of CSF in routine clinical settings. First, mNGS is not available in many hospitals and the cost is usually much higher than target-specific tests. Second, the detection of DNA of a certain pathogen does not necessarily prove that it is responsible for the patient's clinical presentation and features $[23,27]$. In the present study, some patients were positive for EBV but had other more likely aetiologies of their infections. EBV DNA has often been identified together with other microorganisms in CSF [43]. Finally, mNGS cannot detect microorganisms that are not included in microbial genome databases.

Our study has several limitations. Reverse transcription was not performed to construct a DNA library and therefore, RNA viruses could not be detected by mNGS. Next-generation RNA sequencing should be performed in future prospective studies. The number of CNS infections caused by each pathogen were not sufficient to assess the sensitivity and specificity of mNGS for individual pathogens. Conventional microbiological studies used in different centres were not unified. Targeted PCR was not required before mNGS (Supplementary Table 1). Therefore, many common viruses were detected in this study. Finally, we may have underestimated the sensitivity of mNGS of CSF for diagnosing CNS infections by employing strict criteria for a positive mNGS result and using conventional microbiological methods as the gold standard.

\section{Declarations}

\section{Ethics approval and consent to participate}

This study was approved by the institutional review board of Peking Union Medical College Hospital (no. JS-890). Written informed consent was obtained from each patient or their legal surrogate prior to enrolment. The study was performed in accordance with the Declaration of Helsinki.

\section{Consent for publication}


Written informed consent for the use of individual's data for publication was also obtained.

\section{Availability of data and materials}

The datasets used and/or analysed during the current study are available from the corresponding author on reasonable request.

\section{Competing interests}

Author Honglong Wu was employed by company BGI-Tianjin and BGI-Shenzhen. Author Yongjun Li was employed by company BGI-Shenzhen and Vision Medicals. All other authors declare no competing interests.

\section{Funding}

This study was supported by the National Key Research and Development Program of China under Grant (No. 2016YFC0901500); and National Science and Technology Major Project of China under Grant (No.2018ZX10305409).

\section{Authors' contributions}

$\mathrm{HG}$ and SF contributed to the study conception design. All the authors participated in the discussion of the study design. SF, XW, YF, JS, YZ, WZ, XQ, CW, LL, LQ, SW, HJ, HL, WY, WZ, XL, SL, YW, YP, HR, YZ, JW, $B P, L C, W L$, and $H G$ enrolled patients and collected the clinical data. HW, YL and WC performed NGS, bioinformatics analysis, and PCR. SF, HG, XW, and WL analysed the data. SF wrote the first draft of the manuscript after discussions with HG, XW and WL. HW wrote portions of the Methods section. HG, JHC, and WC contributed to manuscript revision. All authors have read and approved the submitted version.

\section{Acknowledgments}

The authors thank the patients for participating in this study.

\section{References}


1. McGill F, Heyderman RS, Panagiotou S, Tunkel AR, Solomon T. Acute bacterial meningitis in adults. Lancet (2016) 388:3036-3047.

2. Venkatesan A, Tunkel AR, Bloch KC, Lauring AS, Sejvar J, Bitnun A, et al. Case definitions, diagnostic algorithms, and priorities in encephalitis: consensus statement of the international encephalitis consortium. Clin Infect Dis (2013) 57:1114-1128.

3. Feigin VL, Nichols E, Alam T, Bannick MS, Beghi E, Blake N, et al. Global, regional, and national burden of neurological disorders, 1990-2016: a systematic analysis for the Global Burden of Disease Study 2016. Lancet Neurol (2019) 18:459-480.

4. Feigin VL, Abajobir AA, Abate KH, Abd-Allah F, Abdulle AM, Abera SF, et al. Global, regional, and national burden of neurological disorders during 1990-2015: a systematic analysis for the Global Burden of Disease Study 2015. Lancet Neurol (2017) 16:877-897.

5. Khatib U, van de Beek D, Lees JA, Brouwer MC. Adults with suspected central nervous system infection: A prospective study of diagnostic accuracy. J Infect (2017) 74:1-9.

6. Hasbun R, Rosenthal N, Balada-Llasat JM, Chung J, Duff S, Bozzette S, et al. Epidemiology of Meningitis and Encephalitis in the United States, 2011-2014. Clin Infect Dis (2017) 65:359-363.

7. Granerod J, Ambrose HE, Davies NW, Clewley JP, Walsh AL, Morgan D, et al. Causes of encephalitis and differences in their clinical presentations in England: a multicentre, population-based prospective study. Lancet Infect Dis (2010) 10:835-844.

8. Glaser CA, Honarmand S, Anderson LJ, Schnurr DP, Forghani B, Cossen CK, et al. Beyond viruses: clinical profiles and etiologies associated with encephalitis. Clin Infect Dis (2006) 43:1565-1577.

9. Gu W, Miller S, Chiu CY. Clinical Metagenomic Next-Generation Sequencing for Pathogen Detection. Annu Rev Pathol (2019) 24:319-338.

10. Wilson MR, Suan D, Duggins A, Schubert RD, Khan LM, Sample HA, et al. A Novel Cause of Chronic Viral Meningoencephalitis: Cache Valley Virus. Ann Neurol (2017) 82:105-114.

11. Mongkolrattanothai K, Naccache SN, Bender JM, Samayoa E, Pham E, Yu G, et al. Neurobrucellosis: Unexpected Answer From Metagenomic Next-Generation Sequencing. J Pediatric Infect Dis Soc (2017) 6:393-398.

12. Fan S, Ren H, Wei Y, Mao C, Ma Z, Zhang L, et al. Next-generation sequencing of the cerebrospinal fluid in the diagnosis of neurobrucellosis. Int J Infect Dis (2017) 67:20-24.

13. Yao M, Zhou J, Zhu Y, Zhang Y, Lv X, Sun R, et al. Detection of Listeria monocytogenes in CSF from Three Patients with Meningoencephalitis by Next-Generation Sequencing. J Clin Neurol (2016) 12:446-451.

14. Fan S, Qiao X, Liu L, Wu H, Zhou J, Sun R, et al. Next-Generation Sequencing of Cerebrospinal Fluid for the Diagnosis of Neurocysticercosis. Front Neurol (2018) 9:471.

15. Guan H, Shen A, Lv X, Yang X, Ren H, Zhao Y, et al. Detection of virus in CSF from the cases with meningoencephalitis by next-generation sequencing. J Neurovirol (2016) 22:240-245. 
16. Wilson MR, Shanbhag NM, Reid MJ, Singhal NS, Gelfand JM, Sample HA, et al. Diagnosing Balamuthia mandrillaris Encephalitis With Metagenomic Deep Sequencing. Ann Neurol (2015) 78:722-730.

17. Wilson MR, Naccache SN, Samayoa E, Biagtan M, Bashir H, Yu G, et al. Actionable diagnosis of neuroleptospirosis by next-generation sequencing. N Engl J Med (2014) 370:2408-2417.

18. Parize P, Muth E, Richaud C, Gratigny M, Pilmis B, Lamamy A, et al. Untargeted next-generation sequencing-based first-line diagnosis of infection in immunocompromised adults: a multicentre, blinded, prospective study. Clin Microbiol Infect (2017) 23:574.e1-574.e6.

19. Naccache SN, Peggs KS, Mattes FM, Phadke R, Garson JA, Grant P, et al. Diagnosis of neuroinvasive astrovirus infection in an immunocompromised adult with encephalitis by unbiased next-generation sequencing. Clin Infect Dis (2015) 60:919-923.

20. Hoffmann B, Tappe D, Hoper D, Herden C, Boldt A, Mawrin C, et al. A Variegated Squirrel Bornavirus Associated with Fatal Human Encephalitis. N Engl J Med (2015) 373:154-162.

21. Brown JR, Morfopoulou S, Hubb J, Emmett WA, Ip W, Shah D, et al. Astrovirus VA1/HMO-C: an increasingly recognized neurotropic pathogen in immunocompromised patients. Clin Infect Dis (2015) 60:881-888.

22. Wilson MR, O'Donovan BD, Gelfand JM, Sample HA, Chow FC, Betjemann JP, et al. Chronic Meningitis Investigated via Metagenomic Next-Generation Sequencing. JAMA Neurol (2018) 75:947955.

23. Brown JR, Bharucha T, Breuer J. Encephalitis diagnosis using metagenomics: application of next generation sequencing for undiagnosed cases. J Infect (2018) 76:225-240.

24. Miao Q, Ma Y, Wang Q, Pan J, Zhang Y, Jin W, et al. Microbiological Diagnostic Performance of Metagenomic Next-generation Sequencing When Applied to Clinical Practice. Clin Infect Dis (2018) 67:S231-s240.

25. Solomon T, Michael BD, Smith PE, Sanderson F, Davies NW, Hart IJ, et al. Management of suspected viral encephalitis in adults-Association of British Neurologists and British Infection Association National Guidelines. J infect (2012) 64:347-373.

26. Tunkel AR, Glaser CA, Bloch KC, Sejvar JJ, Marra CM, Roos KL, et al. The management of encephalitis: clinical practice guidelines by the Infectious Diseases Society of America. Clin Infect Dis (2008) 47:303-327.

27. Granerod J, Cunningham R, Zuckerman M, Mutton K, Davies NW, Walsh AL, et al. Causality in acute encephalitis: defining aetiologies. Epidemiol Infect (2010) 138:783-800.

28. Schlaberg R, Chiu CY, Miller S, Procop GW, Weinstock G. Validation of Metagenomic Next-Generation Sequencing Tests for Universal Pathogen Detection. Arch Pathol Lab Med (2017) 141:776-786.

29. Perlejewski K, Popiel M, Laskus T, Nakamura S, Motooka D, Stokowy T, et al. Next-generation sequencing (NGS) in the identification of encephalitis-causing viruses: Unexpected detection of human herpesvirus 1 while searching for RNA pathogens. J Virol Methods (2015) 226:1-6. 
30. Beck ES, Ramachandran PS, Khan LM, Sample HA, Zorn KC, O'Connell EM, et al. Clinicopathology conference: 41-year-old woman with chronic relapsing meningitis. Ann Neurol (2018) 85:161-169.

31. Zhao W, Wu Y, Li H, Li SY, Fan SY, Wu HL, et al. Clinical experience and next-generation sequencing analysis of encephalitis caused by pseudorabies virus. National Medical Journal of China (2018) 98:1152-1157.

32. Strong MJ, Xu G, Morici L, Splinter Bon-Durant S, Baddoo M, Lin Z, et al. Microbial contamination in next generation sequencing: implications for sequence-based analysis of clinical samples. PLoS Pathog (2014) 10:e1004437.

33. Laurence M, Hatzis $C$, Brash DE. Common contaminants in next-generation sequencing that hinder discovery of low-abundance microbes. PLoS One. (2014) 9:e97876.

34. Kulakov LA, McAlister MB, Ogden KL, Larkin MJ, O'Hanlon JF, et al. Analysis of bacteria contaminating ultrapure water in industrial systems. Appl Environ Microbiol (2002) 68:1548-1555.

35. Hocquet D, Muller A, Bertrand X. What happens in hospitals does not stay in hospitals: antibioticresistant bacteria in hospital wastewater systems. J Hosp Infect (2006) 93:395-402.

36. Verani M, Bigazzi R, Carducci A. Viral contamination of aerosol and surfaces through toilet use in health care and other settings. Am J Infect Control (2014) 42:758-762.

37. Lusk RW. Diverse and widespread contamination evident in the unmapped depths of high throughput sequencing data. PLoS One (2014) 9:e110808.

38. Nguyen TH, Park MD, Otto M. Host Response to Staphylococcus epidermidis Colonization and Infections. Front Cell Infect Microbiol (2017) 7:90.

39. Chew KL, Lee CK, Cross GB, Lum LHW, Yan B, Jureen R. Culture-confirmed cryptococcal meningitis not detected by Cryptococcus PCR on the Biofire meningitis/encephalitis panel ${ }^{\circledR}$. Clin Microbiol Infect (2018) 24:791-792.

40. Ellis JE, Missan DS, Shabilla M, Martinez D, Fry SE. Rapid infectious disease identification by nextgeneration DNA sequencing. J Microbiol Methods (2017) 138:12-19.

41. Grad YH, Lipsitch M, Feldgarden M, Arachchi HM, Cerqueira GC, Fitzgerald M, et al. Genomic epidemiology of the Escherichia coli 0104:H4 outbreaks in Europe, 2011. Proc Natl Acad Sci U S A. (2012) 109:3065-3070.

42. Tang P, Croxen MA, Hasan MR, Hsiao WW, Hoang LM. Infection control in the new age of genomic epidemiology. Am J Infect Control (2017) 45:170-179.

43. Martelius T, Lappalainen M, Palomaki M, Anttila VJ. Clinical characteristics of patients with Epstein Barr virus in cerebrospinal fluid. BMC Infect Dis (2011) 11:281.

\section{Tables}


Table 1. Case definitions and exclusion criteria for encephalitis and meningitis

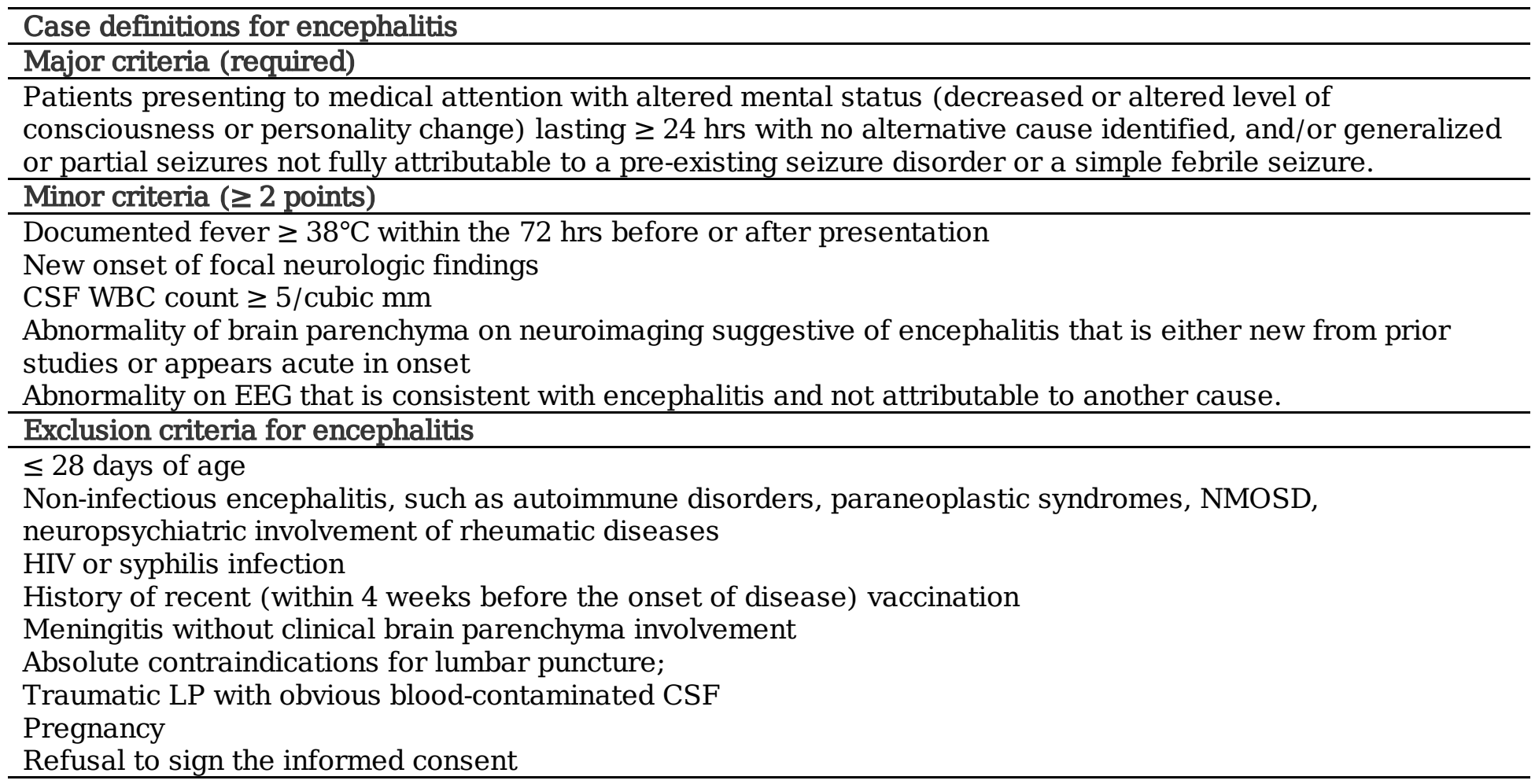

\section{Case definitions for meningitis}

Patients presenting to medical attention with at least two of the four symptoms of headache, fever (documented fever $\geq 38^{\circ} \mathrm{C}$ within the $72 \mathrm{hrs}$ before or after presentation), neck stiffness, decreased level of consciousness (defined by a Glasgow Coma Scale score below 14)

CSF white blood cell count $\geq 5 /$ cubic $\mathrm{mm}$

\begin{tabular}{l} 
Exclusion criteria for meningitis \\
\hline$\leq 28$ days of age \\
HIV or syphilis infection \\
Meningeal malignancy confirmed by CSF cytology \\
Traumatic LP with obvious blood-contaminated CSF \\
Pregnancy \\
Refusal to sign the informed consent \\
\hline
\end{tabular}


Table 2. Performance of mNGS of CSF compared to conventional microbiological studies for the diagnosis of CNS infections

\begin{tabular}{lllll}
\hline & TP $^{*}$ & FP & FN & Etiologic Pathogen \\
\hline Bacteria & & & & \\
\hline Mycobacterium tuberculosis & 14 & 0 & 1 & 15 \\
Listeria monocytogenes & 8 & 0 & 0 & 8 \\
Brucella & 7 & 1 & 1 & 8 \\
Streptococcus pneumoniae & 5 & 0 & 0 & 5 \\
Klebsiella pneumoniae & 3 & 0 & 0 & 3 \\
Streptococcus intermedius & 2 & 0 & 0 & 2 \\
Haemophilus influenzae & 2 & 0 & 0 & 2 \\
Vibrio vulnificus & 1 & 0 & 0 & 1 \\
Staphylococcus hominis & 1 & 0 & 0 & 1 \\
Escherichia coli & 0 & 2 & 0 & 0 \\
Enterococcus faecium & 0 & 2 & 0 & 0 \\
Acinetobacter baumannii & 1 & 2 & 0 & 1 \\
Stenotrophomonas maltophilia & 1 & 1 & 0 & 1 \\
Pseudomonas aeruginosa & 0 & 4 & 1 & 1 \\
Staphylococcus aureus & 0 & 0 & 1 & 1 \\
Staphylococcus haemolyticus & 0 & 0 & 1 & 1 \\
\hline DNA Viruses & & & & \\
\hline Varicella-zoster virus & 17 & 0 & 4 & 21 \\
Herpes simplex virus 1 & 12 & 0 & 1 & 13 \\
Epstein-Barr virus & 12 & 0 & 3 & 6 \\
Cytomegalovirus & 4 & 0 & 0 & 2 \\
Herpes simplex virus 2 & 2 & 0 & 0 & 2 \\
Suid herpesvirus 1 & 2 & 0 & 0 & 2 \\
BK polyomavirus & 1 & 0 & 0 & 0 \\
John Cunningham virus & 1 & 0 & 0 & 1 \\
\hline Fungi & & & & \\
\hline Cryptococcus neoformans & 7 & 0 & 3 & 10 \\
Cryptococcus gattii & 1 & 0 & 0 & 1 \\
\hline Parasites & 5 & 0 & 0 & 5 \\
\hline Taenia solium & 1 & 0 & 0 & 1 \\
Angiostrongylus cantonensis & 110 & 12 & 16 & 114 \\
Totals & & & & \\
\hline & & & & \\
& & & & \\
& & &
\end{tabular}

${ }^{*}$ TP: true-positive; FP: false-positive; FN: false-negative; mNGS: metagenomic next-generation sequencing; CSF: cerebrospinal fluid; CNS: central nervous system

\section{Figures}




\begin{tabular}{|l|l} 
& 11 patients excluded: \\
Systematic vasculitis: 2 patients \\
Rusmusen encephalitis: 1 patient \\
Neuromyelitis optica spectrum disorders: 1 patient \\
Idiopathic hypertrophic pachymeningitis: 1 patient \\
Sjogren's syndrome: 1 patient \\
Primary central nervous system lymphoma: 1 patient \\
Autoinflammatory diseases: 1 patient \\
Primary angiitis of the central nervous system: 1 patient \\
Glioma: 1 patient \\
Neuro-Behcets's syndrome: 1 patient
\end{tabular}

276 patients included

\section{Figure 1}

Flowchart of patient enrolment and exclusion.
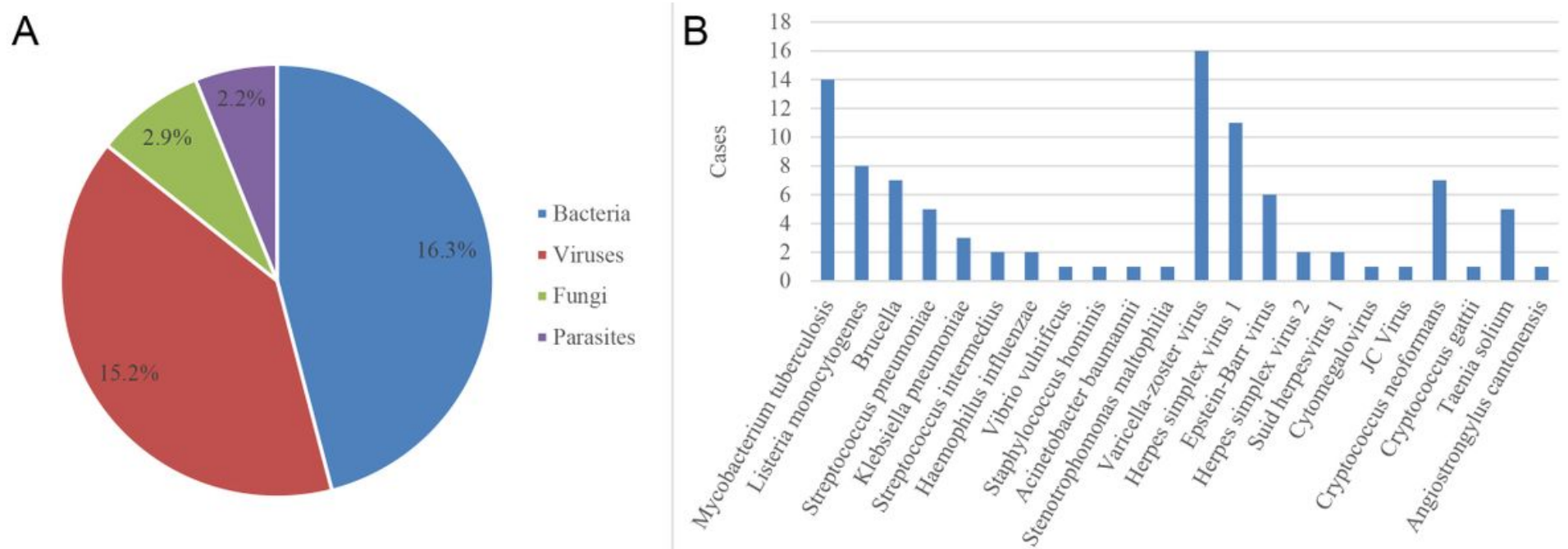

Figure 2

Distribution of causative pathogens in patients with suspected CNS infections initially detected by NGS of CSF. (A) Of the $36.6 \%$ patients diagnosed with NGS of CSF, $16.3 \%$ were diagnosed with bacterial infections, $15.2 \%$ with viral infections, $2.9 \%$ with fungal infections, and $2.2 \%$ with parasitic infections. (B) NGS detected 11 bacterial species, the most common of which were M. tuberculosis $(13.9 \%)$ and $\mathrm{L}$. monocytogenes (7.9\%), 7 viral species, the most common of which were VZV (16.8\%) and HSV1 (11.9\%), 2 fungal species, both of which were Cryptococcus (7.9\%) species, and 2 parasitic species, the most common of which was T. solium (5.0\%). 


\section{Supplementary Files}

This is a list of supplementary files associated with this preprint. Click to download.

- SupplementaryTable320190901.doc

- SupplementaryTable120190901.doc

- SupplementaryTable220190901.doc 